\title{
FATORES PREDITIVOS PARA O DESENVOLVIMENTO DE ÚLCERAS POR PRESSÃO SEGUNDO A ESCALA DE BRADEN EM PACIENTES DE UTI
}

\author{
Cleiciane Vieira de Lima BARROS \\ Rodrigo Cardoso da SILVA ${ }^{2}$ \\ Joyce Morgana Santos ANDRADE ${ }^{3}$ \\ David Antônio Costa BARROS ${ }^{4}$ \\ Ludimila Cristina Souza SILVA 5 \\ Warlley Vieira de LIMA ${ }^{6}$
}

${ }^{1}$ Enfermeira. Especialista em Docência do Ensino Superior. Docente da Faculdade Alfredo Nasser. Mestranda em Enfermagem pela Faculdade de Enfermagem da Universidade Federal de Goiás.

${ }^{2}$ Enfermeiro. Pós-graduando em Enfermagem do Trabalho pelo Instituto Consciência-GO. Email: enf.rodrigo1@gmail.com

${ }^{3}$ Enfermeira.

${ }^{4}$ Enfermeiro. Mestre em Medicina Tropical pelo Instituto de Patologia Tropical e Saúde Pública da Universidade Federal de Goiás. Diretor do Instituto de Ciências da Saúde, Coordenador do curso de Enfermagem e Docente da Faculdade Alfredo Nasser.

${ }^{5}$ Enfermeira. Especialista em Unidade de Terapia Intensiva. Docente da Faculdade Alfredo Nasser. Mestre em Enfermagem. Doutoranda em Ciências da Saúde pela Faculdade de Medicina da Universidade Federal de Goiás.

${ }^{6}$ Farmacêutico.

Recebido em: 22/03/2014 - Aprovado em: 30/06/2014 - Disponibilizado em: 30/07/2014

\section{RESUMO:}

Trata-se de um estudo que sintetizou o conhecimento produzido acerca da utilização da Escala de Braden (EB) na identificação de fatores preditivos para úlcera por pressão (UP) em pacientes críticos. Esta revisão teve como objetivo ressaltar a importância da utilização da EB na determinação dos fatores de risco associados à úlcera por pressão nos pacientes internados em unidades de terapia intensiva, bem como, demonstrar o papel fundamental da equipe de enfermagem, frente à prevenção e tratamento dessas lesões. Foi realizada a revisão bibliográfica, utilizando dados dos indexadores LILACS, SCIELO, MEDLINE além de livros, dissertações e teses, no período 2000 a 2011, em língua portuguesa e inglesa. Em seguida, foi realizada leitura analítica para ordenar as informações e identificar o objeto de estudo. Observou-se com o estudo, que a adoção da EB na determinação e conhecimento dos fatores preditivos para UP nos pacientes institucionalizados, contribui para instituir medidas e um plano de cuidado, visando à redução da incidência destas em unidades que possuem pacientes acamados, e a equipe de enfermagem, exerce grande importância na prevenção, controle e tratamentos dos acometidos. Diante disso, conclui-se que é de grande relevância a realização de estudos que abordem esta temática e evidenciem a necessidade da adoção de escalas como a de Braden na identificação dos riscos e consequente redução da incidência de úlcera por pressão.

Palavras chaves: Úlcera por pressão. Fatores de risco. UTI. Escala de Braden. Paciente crítico.

ABSTRACT:

This is a study that summarized the knowledge produced about the use of the Braden Scale to identify predictive factors for pressure ulcers in critically ill patients. This review aimed to highlight the importance of the use of the Braden scale in determining the risk factors associated with pressure ulcers in patients admitted to intensive care units, as well as demonstrating the key role of the nursing staff, opposite the prevention and treatment of these lesions. The literature review was performed using data from crawlers LILACS, SciELO, MEDLINE, PUBMED plus books, dissertations and theses in the period 2000-2011, in Portuguese and English. Then, analytical reading was performed to sort the information identify the object of study. Was observed with the study, that the adoption of the Braden scale in the determination and knowledge of predictive factors for pressure ulcers in institutionalized patients contributes to institute measures and a plan of care in order to reduce the incidence of these units that have bedridden patients and nursing staff, has great importance in the prevention, control and treatment of affected infants. Therefore, we conclude that it is of great importance to conduct studies that address this issue and reveal the need to adopt scales as Braden identifying risks and consequent reduction in the incidence of pressure ulcers.

Keywords: Pressure ulcers. Risk factors. ICU. Braden scale. Critical patient. 


\section{INTRODUÇÃO}

As Unidades de Terapia Intensiva (UTI) constituem um conjunto de elementos imprescindíveis, destinados à assistência de pacientes em estado crítico, que necessitam constantemente de cuidados, equipamentos e equipe especializada e humanizada. (MINISTÉRIO DA SAÚDE, 1998).

A restrição da mobilidade corporal, de certa maneira, está presente na maioria dos pacientes críticos institucionalizados, incapacitando ou dificultando a sua efetiva movimentação no leito. Isso se deve a inúmeros fatores, como a restrição mecânica, aparelhos ortopédicos e aparelhos gessados, ou ainda, pode estar associado a algum quadro patológico como coma, paralisia, parestesia, sedação, dor, cirurgias e traumas (FERNANDES, 2000).

A UP é uma das complicações pela prolongada imobilização ao leito, acarretando problemas para assistência integral à saúde do paciente e ônus ás instituições de saúde, devido à alta incidência e peculiaridade do tratamento dispensado a esse tipo de lesão e pelo aumento do gasto financeiro decorrente do tratamento por cada paciente acometido, além disso, prorroga o tempo de internação, favorecendo o surgimento de novos agravos (ALBUQUERQUE, 2008).

Desta forma, é fundamental que os profissionais de saúde conheçam todo o processo de fisiopatologia das UP, assim como, tenham como ferramenta de trabalho instituir medidas eficazes na identificação dos fatores de risco para o seu desenvolvimento. (FERNANDES, TORRES, VIEIRA, 2008).

A UP é caracterizada por uma ferida na pele ou no tecido subjacente que na maioria das vezes está localizada em proximidade a uma área de proeminência óssea, ocasionada devido a uma pressão isolada ou podendo ainda estar associada a fatores de risco intrínseco e extrínsecos (FERNANDES, 2000; SILVA, FIGUEIREDO, MEIRELES, 2009; MIYAZAKI, 2009).

Para a identificação dos fatores de risco e consequentemente a melhor conduta a ser dispensada ao paciente, vários autores têm desenvolvido escalas que auxiliam nessa avaliação, como a de Norton, Waterlow, Braden e outras (COSTA, 2003).

A EB, no qual trata essa revisão, foi elaborada por Braden e Bergstron et al., em 1987, como instrumento de avaliação do risco para UP. É composta por seis subescalas: percepção sensorial, atividade, mobilidade, umidade, nutrição, fricção e cisalhamento (COSTA, 2003).

Em uma avaliação sistematizada pelo enfermeiro, faz-se necessário a delimitação de metas, e a elaboração de estratégias e um plano de cuidado, para a resolução do problema, visando eliminar ou minimizar o maior número de fatores de riscos obtidos através da EB com o objetivo de prevenir ou reduzir as UPs nas UTI.

O conhecimento dos fatores de risco 
que influenciam o desenvolvimento de UP é de grande relevância, pois configuram um problema que atinge o paciente fisicamente e psicologicamente, retardando assim sua recuperação. (FERNANDES, 2005). Desta forma, é necessário que os enfermeiros estejam empenhados em aplicar a EB, capacitando a sua equipe na detecção e na prevenção dos fatores de risco. (CREUTZBERG et al, 2011).

Considerando a necessidade da implementação da EB pelo enfermeiro enquanto integrante da equipe multidisciplinar nas UTI e nos serviços de saúde em que existe grande quantidade de acamados, e pela necessidade de estudos que complementem a atuação efetiva do enfermeiro no tratamento e na prevenção de úlceras por pressão, justificamos a relevância deste estudo, que teve como objetivo ressaltar a importância da utilização da EB na determinação dos fatores de risco associados à UP em pacientes de UTI, bem como, associar o papel fundamental da equipe de enfermagem frente à prevenção e tratamento dessas lesões.

\section{MATERIAIS E MÉTODO}

Trata-se de uma revisão bibliografia do tipo descritivo-exploratório e retrospectivo da literatura.

O estudo bibliográfico consiste em explorar toda a literatura existente, obtidas por meio de livros, artigos, dissertação e teses provenientes de bibliotecas tradicionais ou virtuais. $\mathrm{O}$ estudo descritivo-exploratório proporciona maior intimidade e conhecimento a respeito do assunto e objetivos do estudo, criam levantamentos e julgamentos e estabelecem relação entre as variáveis estudadas (GIL, 2002).

Após a definição do tema, levando em consideração a relevância e a exequibilidade do assunto a ser discorrido, utilizou-se a estratégia PICO para facilitar a construção do problema ou pergunta de pesquisa e simplificar o plano de trabalho e auxiliar nos procedimentos seguintes.

Diante disso, foi realizada a busca em bases de dados tradicionais e virtuais em saúde, principalmente nos indexadores: Literatura Internacional em Ciências da Saúde (MEDLINE), Literatura Latino americana em Ciências da Saúde (LILACS), Scientific Electronic Library Online (SCIELO), em língua portuguesa e inglesa, no período de 2000 a 2011, caracterizando o estudo retrospectivo, além de livros, dissertações e teses que se mostraram de relevância para o estudo. Para a coleta de dados os termos utilizados foram combinados com descritores controlados, como os Descritores em Ciência da Saúde (DeCs): Úlcera por pressão, Fatores de risco e Unidade de terapia intensiva, além dos descritores não controlados, como Escala de Braden e Paciente Crítico. Essa medida foi utilizada para aumentar a amostra de 
bibliografia disponível.

Como critérios de seleção foram considerados as obras com dados que abordassem os fatores de risco para UP de acordo com a EB em pacientes de UTI e outras informações específicas relacionadas ao assunto. Em seguida, foi realizada leitura analítica dos títulos e resumos de toda a bibliografia selecionada para ordenar as informações e identificar os objetos de estudo. O passo seguinte, foi a leitura exploratória e interpretativa das publicações, com a finalidade de ressaltar as principais ideias e os dados mais importantes dos estudos considerados para essa revisão.

A seguir, os dados apresentados foram submetidos à análise de conteúdo e posteriormente, os resultados foram discutidos com o suporte de outros estudos, provenientes de revistas científicas e livros, para a construção do relatório final e publicação do trabalho no formato da Associação Brasileira de Normas Técnicas (ABNT).

\section{RESULTADOS E DISCUSSÃO}

Os pacientes identificados como críticos são aqueles que necessitam de cuidados intermitentes e do uso de aparelhos para a manutenção e estabilização dos sinais vitais, possuem diagnóstico grave ou os que foram submetidos a cirurgias que colocam em risco a manutenção da vida. (FERNANDES, 2000)

Em decorrência dessa situação, necessitam de unidades com recursos humanos e materiais especializados e destinados a esse tipo paciente com quadro de saúde grave ou com elevado risco de morte. Necessitam ainda, para o melhor restabelecimento de sua saúde, de uma assistência segura e multidisciplinar. (MINISTÉRIO DA SAÚDE, 1998).

O paciente na UTI está susceptível a inúmeros procedimentos invasivos, como sondagem nasogástrica, enteral, vesical, entubação orotraqueal, bem como, está sujeito a complicações decorrentes desses procedimentos. (FERNANDES, 2005).

$\mathrm{O}$ estado geral comprometido, os constantes procedimentos realizados $\mathrm{e}$ as possíveis complicações que podem advir do seu quadro de saúde, podem aumentar a permanência do paciente na UTI. O longo período de permanência do paciente restrito ao leito o torna mais vulnerável ao desenvolvimento de UP. Sendo assim, o desencadeamento desta é diretamente proporcional à prolongada imobilização (ALBUQUERQUE, 2008).

Esse tipo de lesão é consequência da pressão exercida por alguma área corpórea em alguma superfície por período prolongado, ocasionando a hipóxia celular com a trombose de pequenos vasos, resultando em lesões e em necrose tecidual, em decorrência da falta ou 
baixo suprimento sanguíneo. Geralmente, as áreas corpóreas mais afetadas estão localizadas próximas a proeminências ósseas, como o grande trocanter, tuberosidades isquiáticas, região sacra, calcâneo, maléolo, entre outros (SMELTZER, BARE, 2005).

Condições de idade avançada, obesidade, estado imunológico deficiente, desnutrição, desidratação, edema e áreas com maior transpiração, incontinência urinária e fecal, doença vascular periférica, anemia, hipoalbuminemia, diabetes mellitus, contraturas musculares, lesão medular espinhal, deficiência neurológica ou percepção sensorial, câncer metastático, esclerose múltipla, além de fatores extrínsecos como pressão, cisalhamento e fricção, constituem um conjunto relevante de fatores de risco para o desencadeamento de UP, ou seja, fatores intrínsecos e extrínsecos estão envolvidos com o surgimento dessas lesões (FERNANDES, 2000; SILVA, FIGUEIREDO, MEIRELES, 2009).

A percepção sensorial é importante, pois quando a mesma é afetada, o paciente diminui a capacidade de identificar desconforto ou a dor. Esta diminuição é decorrente muitas vezes de sedação, uso de anestésicos, analgésicos e o estado patológico (FERNANDES, 2000) e somam-se a estas, outras condições como, coma, uso de trações ortopédicas e recuperação de grandes cirurgias que desencadeiam a falta de estímulo para que o indivíduo se mova em busca de alivio (ROGENSKI, 2011).

$\mathrm{O}$ estado nutricional deficiente expõe os tecidos a lesões. Portanto, é de total relevância que o organismo esteja adequadamente nutrido, para assim, responder de forma significativa às agressões externas. A falta de algumas vitaminas interfere no processo de produção de colágeno e elastina favorecendo assim a fragilidade capilar. (FERNANDES, 2000).

$\mathrm{Na}$ UTI é comum encontrarmos pacientes com distúrbios hematológicos, além de outros fatores que desencadeiam úlceras por pressão, sendo de total importância uma equipe multiprofissional capacitada para identificar tais riscos (FERNANDES, 2000).

A umidade é um fator de risco relevante para o desenvolvimento de UP (COSTA, 2003). A umidade é considerada a subescala de maior importância na EB, seguido pela percepção sensorial em pacientes acamados em UTI (ROGENSKI, 2011).

Outro fator relevante é a fricção, quando duas superfícies se movimentam uma sobre a outra, acarretando na destruição dos tecidos. Esta pode ser causada por mudanças de decúbito inadequadas (FERNANDES, 2000). Em pacientes na UTI a ocorrência de fricção é muito comum, em decorrência dos mesmos não conseguirem se movimentar sozinhos, os profissionais muitas vezes os arrastam sobre a superfície do leito (COSTA, 2003).

A força de cisalhamento ocorre 
quando o paciente desliza na cama, e a pele permanece imóvel (SILVA, FIGUEIREDO, MEIRELES, 2009). As forças de fricção e cisalhamento ocorrem simultaneamente, quando o paciente não se posiciona de forma adequada no leito e desliza o corpo sobre o mesmo (COSTA, 2003). A presença do cisalhamento ocasiona danos aos tecidos, sendo o tecido muscular o mais acometido e a área do corpo mais afetada é a região sacral (FERNANDES, 2000).

Segundo estudo realizado, os fatores extrínsecos que mais contribuíram para o desenvolvimento de UP, foram o colchão inadequado, paciente em um mesmo decúbito por mais de 2 horas e roupas de cama mal esticadas (FERNANDES, TORRES, VIEIRA, 2008). Indicando falha em cuidados básicos exercidos pela equipe de enfermagem, essa negligencia, pode estar relacionada a situações como inadequação da quantidade de profissionais no serviço e maior tempo dispensado a cuidados de maior complexidade e que exigem maior atenção da equipe, entretanto, ações simples de cuidado não podem ser menosprezadas.
A alta incidência e prevalência desse tipo de lesão, muitas vezes, podem estar associadas ao ato negligente da equipe de enfermagem que não realiza conforme necessário a mudança de decúbito do paciente acamado, visto que o mesmo possui mobilidade física reduzida ou ausente, gerando assim, no primeiro momento, uma hiperemia local, considerada o primeiro estádio da lesão. Esta, se não for tratada, poderá progredir para o segundo estádio que atinge a derme, podendo acarretar ainda outras complicações ao paciente. (SILVA, FIGUEIREDO, MEIRELES, 2009).

As UP são classificadas em estádios que vão de I a IV, conforme mostra a tabela 1, de acordo com o proposto pelo "National Pressure Ulcer Advisory Panell” (NPUAP), que é um órgão americano responsável pela sistematização de diretrizes para a prevenção, levantamento de dados estatísticos, tratamento e condutas relacionadas à UP (CAMARGO, 2006). Ainda, a tabela aqui apresentada, foi revisada pela "Agency for Health Care Policy and Research" (AHCBR).

\section{Tabela I - Estádios das úlceras por pressão, proposto pelo NPUAP e revisada pelo AHCBR} (1989).

\begin{tabular}{|l|c|}
\hline Estádio I & $\begin{array}{c}\text { Pele intacta, com presença de hiperemia local que mesmo após a retirada da pressão não } \\
\text { embranquece. Estas podem ser difíceis de serem detectadas em pessoas de tonalidades escuras de pele. }\end{array}$ \\
\hline Estádio II & Perda parcial da derme e epiderme sem a presença de esfacelo pode apresentar-se brilhante ou seca. \\
\hline & $\begin{array}{c}\text { Perda total da espessura da pele, sem exposição de músculo, osso ou tendão. Pode haver a presença de } \\
\text { Estádio III }\end{array}$ \\
\hline
\end{tabular}




\begin{tabular}{|l|l|}
\hline Estádio IV & $\begin{array}{c}\text { Perda total da espessura da pele, atingindo músculo, osso ou tendão. O esfacelo pode estar presente, a } \\
\text { profundidade varia de acordo com a localização anatômica. }\end{array}$ \\
\hline
\end{tabular}

Fonte: ROGESKI, 2011. Adaptado.

Estudo realizado no Brasil por Costa (2003) demonstrou que 37,7\% dos pacientes internados na UTI, desenvolveram UP, sendo em média, 2,95 úlceras por pacientes, em diferentes localidades e estádios. Pesquisa realizada com pacientes também internados em UTI, no interior de São Paulo, mostra que dos 48 pacientes acompanhados, 30 desenvolveram UP, sendo a taxa de incidência de 62,5\%. (FERNANDES, CALIRI, 2008).

A EB, conforme demonstrado na tabela 2, foi elaborada como instrumento de avaliação preditiva do risco para UP (COSTA, 2003). Esta oferece seis parâmetros de alto risco para a UP, sendo constituídos das seguintes subescalas: 1- percepção sensorial, 2-umidade, 3- atividade, 4- mobilidade, 5nutrição, 6- fricção e cisalhamento. Cada subescala tem pontuação que varia de um a quatro, exceto fricção e cisalhamento, que é pontuada de um a três. A somatória total tem valor mínimo de seis e máximo de vinte e três pontos. (BLANES et al, 2004; FERNANDES; CALIRI, 2008). Sendo que uma pontuação abaixo de onze indica risco elevado, entre doze e quatorze, risco moderado e de quinze a dezesseis, risco mínimo. (SOUZA, SANTOS, SILVA, 2006).

O tratamento de UP deve contemplar uma elaboração de planos de cuidados e o registro da conduta terapêutica, abordando a classificação, localização, tamanho, aspecto do leito da ferida, dor, drenagem e temperatura. O tratamento inicial da úlcera por pressão pode envolver limpeza da ferida, utilização de curativos, desbridamento e em alguns casos pode ser necessário cirurgia reparadora (SILVA; FIGUEIREDO; MEIRELES, 2009).

Com o surgimento de novas tecnologias, muito se tem contribuído para o aperfeiçoamento do tratamento de UP, o que favorece a melhor qualidade da assistência prestada ao paciente, sendo necessário que o enfermeiro saiba usufruir dos avanços tecnológicos de forma satisfatória. 
Tabela II - Escala de Braden adaptada para a língua portuguesa

\begin{tabular}{|c|c|c|c|c|}
\hline Percepção sensorial: & 1. Totalmente limitado. & 2. Muito limitado. & 3. Pouco limitado. & 4. Sem limitação. \\
\hline Atividade física: & 1. Acamado. & 2. Restrito a cadeira. & 3. Caminha pouco. & $\begin{array}{l}\text { 4. Caminha } \\
\text { Frequentemente. }\end{array}$ \\
\hline Umidade da pele: & $\begin{array}{l}\text { 1. Completamente } \\
\text { úmida. }\end{array}$ & 2. Muito úmida. & 3. Ocasionalmente úmida. & $\begin{array}{l}\text { 4. Raramente } \\
\text { úmida. }\end{array}$ \\
\hline Nutrição: & 1. Muito insuficiente. & $\begin{array}{l}\text { 2. Talvez } \\
\text { inadequada. }\end{array}$ & $\begin{array}{l}\text { 3. Suficientemente } \\
\text { adequada. }\end{array}$ & $\begin{array}{l}\text { 4. Excelentemente } \\
\text { Adequada. }\end{array}$ \\
\hline $\begin{array}{l}\text { Fricção e cisalha- } \\
\text { mento: }\end{array}$ & 1. Existe o problema. & $\begin{array}{l}\text { 2. Problema } \\
\text { potencial. }\end{array}$ & 3. Não há o problema. & \\
\hline
\end{tabular}

Fonte: PARANHOS; SANTOS, 1999. Adaptado.

Por outro lado, os tratamentos de UP, possuem custo elevado para o sistema de saúde, são dolorosos e desagradáveis ao paciente e, além disso, podem provocar alterações psicológicas, emocionais e outras complicações decorrentes do processo de tratamento e da própria lesão (COSTA, 2003; MEDEIROS; LOPES; JORGE, 2009). O que evidencia a necessidade de se prevenir o dano antes que aconteça.

\section{CONSIDERAÇÕES FINAIS}

Diante do levantamento bibliográfico realizado, evidenciou-se a necessidade da aplicação de escalas para mensurar quantitativamente o risco de o paciente acamado desenvolver uma UP. Pode-se afirmar que a UP é um fator condicionante na recuperação do paciente de UTI, pois o surgimento de uma lesão desencadeia uma série de eventos que podem retardar a recuperação deste.

Ressalta-se que fatores extrínsecos e intrínsecos podem contribuir para desencadeamento de UP. Portanto, através da aplicação da EB de forma adequada pelos profissionais de saúde, é possível identificar estes fatores e enumerá-los. Quanto maior o escore, maior é o risco de desenvolver UP, devendo a equipe de enfermagem, ter uma atenção especial para estes riscos que foram identificados com escores elevados, pois são os profissionais que mais tempo permanecem junto aos pacientes.

É inadmissível que o paciente acamado desenvolva uma lesão em consequência da negligência do cuidado. O desenvolvimento de UP nos pacientes é de total responsabilidade da equipe de enfermagem. Devendo estes profissionais 
implementarem e executarem ações simples que promovam o afastamento dos fatores preditores de UP.

Portanto, prevenir uma UP é desenvolver o papel da enfermagem com qualidade, segurança, eficiência, dedicação e humanidade. Essas lesões, além de provocarem danos psicológicos, dor e sofrimento ao paciente de UTI e seus entes, prolongam o tempo de internação e o torna mais vulnerável a outros agravos decorrentes da perda da integridade da pele e do próprio ambiente ao qual está inserido. Diante disso, é notório a necessidade de novos estudos que

\section{REFERÊNCIAS}

\section{ALBUQUERQUE, A. M. Medidas}

preventivas para úlcera por pressão no centro de terapia intensiva: conhecimento e prática dos enfermeiros. Dissertação (Mestrado em Enfermagem). Centro de Ciências da Saúde. Universidade Federal da Paraíba; João

Pessoa; PB. 2008. Disponível em: www.ccs.ufpb.br/ppgeold/. Acesso em: dezembro de 2011.

BLANES, L.; DUARTE, I.S.; CALIL, J. A.; FERREIRA, L. M. Avaliação clínica e epidemiológica das úlceras por pressão em pacientes internados no hospital São Paulo. Revista Assoc. Med. Bras. [online], vol. 50, n. 1, 2004. Disponível em: http://www.scielo.br. Acesso em: outubro de 2011. abordem esta importante temática, e que remeta ao conhecimento de medidas preventivas através do conhecimento dos principais fatores de risco intrínsecos e extrinsecos para o surgimento das UP.

Assim sendo, conclui-se que a prevenção é sempre a melhor forma de reduzir a incidência e prevalência de UP, minimizar o sofrimento paciente e de sua família, reduzir os custos com o tratamento, o tempo de internação e os problemas psicológicos, físicos e sociais advindos da lesão.

\section{BRASIL. MINISTÉRIO DA SAÚDE.}

Portaria nº 466, de 04 de junho de 1998.

Disponível em:

http://www.anvisa.gov.br/legis/portaria/466

98.htm. Acesso em: setembro de 2011.

CAMARGO, S.M.P.L.O. Estudo combinado do ultra-som pulsado de baixa intensidade e da papaína na cicatrização de úlcera por pressão no atendimento domiciliar.

Dissertação (Mestrado em Bioengenharia). Escola de Engenharia de São Carlos Faculdade de Medicina de Ribeirão Preto Instituto de Química de São Carlos da Universidade de São Paulo; 2006. Disponível em: www.teses.usp.br/teses. Acesso em: dezembro de 2011. 
COSTA, I. G. Incidência de úlcera de pressão e fatores de risco relacionados em pacientes de um centro de terapia intensiva. Escola de Enfermagem Ribeirão Preto da Universidade de São Paulo, 2003. Disponível em: http://www.eerp.usp.br/projetos/ Acesso: Dezembro de 2012.

COSTA, I.G. Incidência de úlcera de pressão e fatores de risco relacionados em pacientes de um centro de intensiva. Dissertação (Mestrado em Enfermagem). Escola de Enfermagem de Ribeirão Preto da Universidade de São Paulo; Ribeirão Preto; 2003. Disponível em: www.teses.usp.br/teses. Acesso em: dezembro de 2011.

CREUTZBERG, M.; AGUILERA, N. C. F.; CARDOSO, P. C.; BARBOSA, T. L.; CEOLIN, L. D.; STEIN, K.; URBANETTO, J. S. Fatores de risco para úlceras de pressão em idosos de unidade de terapia intensiva. Revista Enfermagem em foco. [impresso], vol. 2, n. 2, p.133-136, Maio, 2011.

FERNANDES N.C.S.; TORRES G.V.; VIEIRA D. Fatores de risco e condições predisponentes para úlcera de pressão em pacientes de terapia intensiva. Rev. Eletr. Enf., Vol. 10, n. 3, 2008. Disponível em: www.fen.ufg/fen revista. Acesso em: outubro de 2011.

FERNANDES, L. M.; CALIRI, M.H.L. L. M. Uso da escala de Braden e de Glasgow para identificação do risco para úlcera depressão em pacientes internados em centro de terapia intensiva. Revista Latino Americana de Enfermagem, vol. 16, n. 6, 2008. Disponível em: http://www.scielo.br. Acesso em: março de 2012.

FERNANDES, L. M.; Úlcera de pressão em pacientes críticos hospitalizados: Uma revisão integrativa da Literatura. Dissertação (Mestrado em Enfermagem). Escola de Enfermagem de Ribeirão Preto da Universidade de São Paulo; Ribeirão Preto; 2000. Disponível em: www.teses.usp.br/teses. Acesso em: dezembro de 2011.

FERNANDES, N. C. S. Úlceras de pressão: um estudo com pacientes de unidade de terapia intensiva. Dissertação (Mestrado em Enfermagem). Centro de Ciências da Saúde, da Universidade Federal do Rio Grande do Sul; Rio Grande do Sul; 2005. Disponivel em: http://repositorio.ufrn.br:8080/jspui/handle/1/ 9261. Acesso em: dezembro de 2011.

FERNANDES, N. C. S.; TORRES, G. V.; VIEIRA, D. Fatores de risco e condições predisponentes para úlcera de pressão em pacientes de terapia intensiva. Rev. Eletr. Enf., vol.10, n. 3, 2008. Disponível em: www.fen.ufg.br/fen_revista. Acesso em: março de 2012.

GIL, Antônio Carlos. Como elaborar projetos de pesquisa. São Paulo: Ed. Atlas, 2002. MEDEIROS, A. B. F.; LOPES, C. H. A. F.; JORGE, M. S. B. Análise da prevenção e tratamento das úlceras por pressão por enfermeiros. Rev. Esc. Enferm., vol. 43, n. 1, 
2009. Disponível em: http://www.scielo.br. Acesso em: 29 de outubro de 2011.

MIYAZAKI, M. Y. Conhecimento das recomendações para a prevenção da úlcera por pressão pela equipe de enfermagem de um hospital universitário. Dissertação (Mestrado em Enfermagem). Escola de Enfermagem de Ribeirão Preto da Universidade de São Paulo, Ribeirão Preto, 2009. Disponível em: www.teses.usp.br/teses. Acesso em: dezembro de 2011.

\section{NATIONAL PRESSURE ULCER} ADVISORY PANEL (NUPUAP). Disponível em: http://www.npuap.org/._ Acesso em: outubro de 2011.

PARANHOS, W.Y.; SANTOS, V.L.C.G.

Avaliação de risco para úlceras de pressão por meio da Escala de Braden, na língua portuguesa. Revista da Escola de Enfermagem da USP, vol. 33, n. esp., 1999. Disponível em: www.ee.usp.br/reeusp/upload/pdf/799.pdf.

Acesso em: setembro de 2011

ROGENSKI, N. M B. Avaliação da Implementação de um protocolo de prevenção de úlceras por pressão. 2011. Tese (Doutor em Ciências). Escola de Enfermagem da Universidade de São Paulo; São Paulo; 2011. Disponível em: www.teses.usp.br/teses.
ROGENSKI, N. M. B.; SANTOS, V. L. C. G. Estudo sobre a incidência de úlceras por pressão em um hospital universitário. Revista Latino Americana de Enfermagem, Ribeirão Preto, v.13, n. 4, 2005. Disponível em: http://www.scielo.br. Acesso em: outubro de 2011.

SILVA, D. P.; BARBOSA, M. H.; ARAÚJO, D.F.; LORENA, P.O.; ADRIANA, F. M. Úlcera por pressão: avaliação de fatores de risco em pacientes internados em um hospital universitário. Rev. Eletr. Enf., v. 3, n. 1, 2011. Disponível em: www.fen.ufg.br/fen_revista. Acesso em: outubro de 2011.

SILVA, R. C. L. S.; FIGUEIREDO. N. M. A.; MEIRELES. I. B. Feridas: fundamentos e atualizações em enfermagem. 2. ed. e rev. e amp. São Caetano do Sul: Yendis, 2009.

SMELTZER, S.C.; BARE, B.G et al. Brunner e Suddarth, tratado de enfermagem médicocirurgião. Rio de Janeiro: Ed Guanabara Koogan, 2005.

SOUSA, C. A.; SANTOS, I.; SILVA, L. D. Aplicando recomendações da Escala de Braden e prevenindo úlceras por pressãoevidências do cuidar em enfermagem. Revista Brasileira de Enfermagem, vol. 59, n.3, 2006. Disponível em: http://www.scielo.br. Acesso em: outubro de 2011.

Acesso em Janeiro de 2012. 